\title{
TMS Member Projects Receive First Wave of DMREF Funding
}

\section{By Lynne Robinson}

In support of the U.S. Materials Genome Initiative (MGI) the National Science Foundation (NSF) recently announced its first awards for the Designing Materials to Revolutionize and Engineer our Future (DMREF) program.

The NSF Mathematical and Physical Sciences (MPS) and Engineering (ENG) directorates invested a total of just over \$12 million for 22 grants in support of 14 DMREF projects that target the MGI's goal of significantly reducing the current time and cost of transitioning materials breakthroughs from the laboratory to the marketplace. The DMREF's approach to accomplishing this involves the development of new physically based and verified computational tools to accelerate the discovery, development, and property optimization of new materials and systems.

TMS congratulates its following members whose projects have received one of these prestigious grants:

\section{Multi-Scale Fundamental Investigation of Sintering Capolungo, Georgia Tech Research Corporation} Anisotropy

Principal Investigators: Eugene

Olevsky, San Diego State University;

Rajendra Bordia, University of

Washington

The development of a new, integrated, multi-scale approach incorporating modeling and experimentation on sintering-induced deformation processes, taking into account anisotropy phenomena, is the focus of this project. The work will encompass the study of the complex interplay between processing conditions and anisotropic microstructure-constitutive properties, providing fundamental, basic knowledge and a novel, practical approach to optimizing the manufacture of advanced ceramic and metal systems with programmable macroscopic characteristics and microstructure, including multilayered solid oxide fuel cells.

Discovery, Development, and Deployment of High Temperature Coating/Substrate Systems Principal Investigator: Tresa Pollock, University of California, Santa Barbara

This project engages an engineering and computer science team to develop a fundamental framework for the design of new multilayered materials systems for energy-efficient power generation and aircraft propulsion.

\section{Multi-Scale Modeling and} Characterization of TwinningInduced Plasticity and Fracture in Magnesium Alloys

Principal Investigators: Haitham El

Kadiri, Mississippi State University; Sean Agnew, University of Virginia; Co-Principal Investigator: Laurent

The goal of this collaborative effort is to identify fundamentally validated mean-field and full-field models capable of predicting failure in magnesium alloys. These models will greatly aid efforts to render lightweight magnesium alloys "formable" and "crushable," so that society can exploit their performance and efficiency benefits in safety critical applications.

\section{Nitride Discovery - Creating the Knowledge Base for Hard Coating Design}

Principal Investigator: Daniel Gall, Rensselaer Polytechnic Institute
The primary objective of this research program is to develop a method for determining the intrinsic physical properties of transition metal nitrides. By providing a systematic understanding of the fundamental properties of all transition metal nitrides, based on their electronic structure, this knowledge base has the potential to help transform the evolutionary trial-and-error development of protective coatings into a "coatings-by-design" approach.

First-Principles Based Design of Spintronic Materials and Devices Co-Principal Investigator: Subhadra Gupta, University of Alabama

The focus of this project is to provide an improved understanding of half-metals and magnetic materials. Of particular emphasis is how to relate "first-principles" calculations to experimentally accessible and technologically relevant materials and device parameters.

\section{A Fundamental Approach to Study the Effect of Structural and Chemical Composition in Functionalized Graphene Materials Co-Principal Investigator: Jiaxing Huang, Northwestern University}

The central goal of this project is to establish a paradigm shift in materials design by combining theory, modeling, and experimentation in a multiscale and synergistic manner to maximize the strength and toughness of nanocomposite materials that emulate the performance of hierarchically assembled structures inspired by nature, using graphene oxide.

To read the full descriptions of these projects, as well as the other DMREF-funded projects, go to http://www.nsf.gov/news/news_summ. jsp?cntn_id=125712\& 